\title{
CÁC VẤN ĐỀ LIÊN QUAN ĐẾN VIỆC XÂY DỰNG HỆ QUY CHIẾU KHÔNG GIAN QUỐC GIA
}

\author{
PGS. TSKH. HÀ MINH HÒA \\ Viện Khoa học Đo đạc và Bản đồ
}

\section{Tóm tắt:}

Bài báo khoa học này nghiên cứu vấn đề mấu chốt để xây dụng hệ quy chiếu không gian quốc gia - đó là việc xây dựng mô hình Quasigeoid quốc gia độ chính xác cao. Việc giải quyết vấn đề này liên quan đến hàng loạt bài toán khoa học - kỹ thuật phức tạp bao gồm xây dựng mạng lưới GNSS dựa trên đồng thời các công nghệ GPS/GLONASS, xử lý toán học mạng lưới độ cao hạng I, II quốc gia trong hệ độ cao dựa trên mặt Geoid cục bộ Hòn Dấu để xác định các giá trị thế năng trọng trường thực của các mốc độ cao hạng I, II quốc gia, hiệu chỉnh các hệ số khai triển điều hòa của mô hình trọng trường Quả đất EGM dựa trên các dữ liệu đo trọng lực chi tiết trên lãnh thổ quốc gia và các giá trị thế năng trọng trường thực của các mốc độ cao hạng I, Il quốc gia, bình sai ghép nối mạng lưới GNSS vào hệ quy chiếu không gian quốc gia. Bài báo cũng chỉ ra rằng khi hiệu chỉnh các hệ số khai triển điều hòa của mô hình trọng trường Quả đất EGM để nhận được dị thường độ cao trọng lực với độ chính xác cao hơn $0.08 \mathrm{~m}$ là một trong những cơ sở khoa học quan trọng để đạt được độ chính xác của mô hình Quasigeoid ở mức $0.04 \mathrm{~m}$.

\section{1. Đặt vấn đề}

$\mathrm{H}$ ệ tọa độ động lực hay hệ quy chiếu không gian động lực thực chất là hệ tọa độ không gian Quả đất, nhưng hệ tọa độ này có đặc điểm cơ bản sau: gốc của hệ tọa độ và vị trí của các điểm được xác định trong hệ tọa độ này luôn thay đổi theo thời gian theo những quy luật toán học xác định. Khung quy chiếu Quả đất quốc tế ITRF (International Terrestrial Reference Frame) là hệ quy chiếu không gian động lực quốc tế với gốc của hệ quy chiếu trùng với tâm vật chất của Quả đất. Tuy nhiên, do tâm vật chất của Quả đất luôn chuyển động với tốc độ $1 \mathrm{~mm} / 1$ năm, nên ITRF chỉ được xác định vào một thời điểm chuẩn xác định và sau một vài năm lại phải định vị lại gốc của nó vào tâm vật chất Quả đất. Như vậy, vị trí không gian của một điểm sẽ khác nhau trong các ITRF khác nhau.Mặt khác, do chuyển dịch địa động lực của vỏ Trái đất, của các mảng kiến tạo, nên trong một ITRF xác định, vị trí không gian của các điểm được xác định trong ITRF này cũng thay đổi theo thời gian. Tổ chức Dịch vụ GNSS quốc tế (International GNSS Service) có trách nhiệm thường xuyên định vị lại gốc của ITRF vào tâm vật chất của Quả đất và xác định các tốc độ chuyển dịch không gian của các điểm (các trạm thu tín hiệu GNSS (Global Navigation Satellite System)) thuộc mạng lưới IGS. Hệ quy chiếu không gian quốc gia được xây dựng dựa trên mạng lưới GNSS quốc gia được xử lý trong ITRF được gọi là hệ tọa độ động lực quốc gia hoặc hệ quy chiếu không gian động lực quốc gia, hay đơn giản hơn là hệ quy chiếu không gian quốc gia.

Người phản biện: TS. Nguyễn Đình Thành 
Hệ quy chiếu không gian quốc gia được xây dựng để thống nhất các hệ quy chiếu tọa độ mặt phẳng quốc gia và hệ độ cao quốc gia trong hệ thống quy chiếu không gian quốc gia thống nhất. Để bảo tồn các tọa độ phẳng của hệ quy chiếu tọa độ mặt phẳng quốc gia, các điểm cơ sở của hệ quy chiếu không gian quốc gia phải trùng với các điểm thiên văn trắc địa hạng I, II quốc gia. Hệ NSD83 của Mỹ là hệ tọa độ không gian quốc gia. 38 nước châu Âu với khoảng gần 100 các hãng, các trường Đại học tổng hợp đã tham gia vào mạng Iưới thường trực GNSS EUREF (EUropean Reference Earth Frame) bao gồm 218 trạm GNSS thường trực, trong đó 80 trạm GPS/GLONASS, 87 trạm IGS, và đã xây dựng hệ quy chiếu Quả đất châu Âu ETRS89 vào năm 1989 dựa trên ellipsoid quy chiếu GRS80 (Ihde J., Bruyninx C. (2008)). Vương quốc Anh vào năm 2002 đã kết nối các mạng lưới tam giác quốc gia với ETRS89 và đã xây dựng được hệ tọa độ không gian địa tâm OS Net (Ordnance Survey Network) của nước Anh. Mô hình Geoid OSGM02 của nước Anh có độ chính xác ở mức $\pm 2 \mathrm{~cm}$. Trong tài liệu (Sánchez L., Martisnez W. (2001)) đã thông báo rằng hệ tọa độ mặt phẳng Bogota của Colombia được IGAC xây dựng từ năm 1936 với các mạng lưới trắc địa quốc gia truyền thống bao gồm 11.000 điểm hạng I, II. Cùng với việc phát triển SIRGAS vào những năm 1994, 1996 và 1997 IGAC (the Instituto Geográfico Agustín Codazzi) đã xây dựng Khung quy chiếu trắc địa MAGNA (MArco Geocétrico Nacional de ReferenciA) dựa trên việc xây dựng mạng lưới GPS phủ trùm Colobia bao gồm 56 điểm được kết nối với hệ thống SIRGAS2000 và ITRF và sử dụng ellipsoid GRS80. Khung quy chiếu trắc địa MAGNA đảm bảo việc xác định vị trí mặt phẳng ở mức $\pm 2 \mathrm{~cm}$ và độ cao trắc địa ở mức $\pm 6 \mathrm{~cm}$.

Một số ví dụ nêu ở trên phản ánh một thực tế trong xu hướng phát triển hiện đại của Trắc địa cao cấp - đó là xây dựng hệ quy chiếu không gian quốc gia. Ở Việt Nam, trong công trình (Hà Minh Hòa, Đặng Hùng Võ, Phạm Hoàng Lân, Nguyễn Ngọc Lâu (2005)) đã luận chứng các cơ sở khoa học để xây dựng hệ quy chiếu không gian quốc gia. Tuy nhiên, yêu cầu xác định hệ quy chiếu không gian phải đảm bảo để sai số tương đối của mỗi thành phần tọa độ (các tọa độ không gian $\mathrm{X}, \mathrm{Y}, \mathrm{Z}$ hoặc các tọa độ ellipsoid $\mathrm{B}, \mathrm{L}, \mathrm{H}$ ) phải đạt ở mức $10^{-9}$ (Augath W., Ihde J. (2002)).

Trong thực tế ứng dụng công nghệ GNSS (Global Navigation Satellite System), độ chính xác xác định các tọa độ trắc địa $\bar{B}, \bar{L}$ của điểm quan tâm rất cao khi xử lý các dữ liệu đo GNSS trong ITRF. Trong trường hợp này, chúng ta đánh giá độ chính xác có thể đạt được của độ cao trắc địa $\bar{H}$ của điểm. Để đánh giá ước tính độ chính xác của độ cao trắc địa $\bar{H}$ của điểm thuộc mạng lưới GNSS được xử lý trong ITRF, chúng ta sử dụng điều kiện $\mathrm{m}_{\mathrm{X}}=\mathrm{m}_{\mathrm{Y}}=\mathrm{m}_{\mathrm{Z}}=\frac{M_{X Y Z}}{\sqrt{3}}$, ở đây $\mathrm{M}_{X Y Z}$ - sai số vị trí không gian của điểm được đặc trưng bởi độ chính xác của lịch vệ tinh chính xác của Tổ chức IGS và được cho trong ITRF. Khi đó, sai số trung phương của độ cao trắc địa $\bar{H}$ được đánh giá theo công thức (Hà Minh Hòa và nnk (2012a)):

$$
m_{\bar{H}}=\frac{M_{X Y Z}}{\sqrt{3} \cdot \cos B} .
$$

Khi đó sai số tương đối của các thành phần của các tọa độ không gian được đánh giá 


\section{Nghiên cúu}

theo công thức:

$$
\frac{m_{X}}{R}=\frac{m_{Y}}{R}=\frac{m_{Z}}{R}=\frac{m_{\bar{H}} \cdot \cos B}{R}=\frac{M_{X Y Z}}{\sqrt{3} \cdot R},
$$

Ở đây $\mathrm{R}$ - bán kính trung bình của Quả đất.

Với $\mathrm{R}=6356752.314 \mathrm{~m}$ và độ chính xác Lịch vệ tinh chính xác $\mathrm{M}_{\mathrm{XYZ}}=2.5 \mathrm{~cm}$ trong ITRF, từ (1) chúng ta có $\frac{m_{X}}{R}=\frac{m_{Y}}{R}=\frac{m_{Z}}{R}=\frac{M_{X Y Z}}{\sqrt{3} \cdot R}=2 \cdot 27.10^{-9}$ và trên lãnh thổ Việt Nam, sai số tương đối của độ cao trắc địa $\bar{H}$ được đánh giá theo công thức:

$$
\frac{m_{\bar{H}}}{R}=(2.29-2.49) \cdot 10^{-9} .
$$

Như vậy, chỉ khi xử lý mạng lưới GNSS trong ITRF, chúng ta mới đáp ứng được yêu cầu của việc xây dựng hệ quy chiếu không gian. Trong khi đó, đối với hệ quy chiếu không gian quốc gia, độ chính xác của độ cao trắc địa $H$ của điểm được xác định theo công thức $m_{H}=\sqrt{m_{H^{\gamma}}^{2}+m_{\zeta}^{2}}$, ở đây $m_{H^{\gamma}}$ - sai số trung phương của độ cao chuẩn, còn $m_{\zeta}$ - sai số trung phương của dị thường độ cao. Độ chính xác của độ cao chuẩn hạng I $m_{H^{\gamma}}$ thường ở mức $4 \mathrm{~cm}$ so với điểm gốc của hệ độ cao quốc gia, còn độ chính xác xác của độ cao chuẩn hạng $\| m_{H^{\gamma}}$ thường ở mức $5 \mathrm{~cm}$. Với bán kính trung bình của Quả đất $\mathrm{R}=$ $6356752.314 \mathrm{~m}$, chúng ta sẽ đánh giá sai số tương đối $\frac{m_{H}}{R}$ theo các giá trị $m_{\zeta}$ và nhận được các kết quả ở bảng 1 ở dưới đây.

Bảng 1

\begin{tabular}{|c|c|c|}
\hline$m_{\zeta}(\mathrm{m})$ & $m_{H}(\mathrm{~m})$ & $\frac{m_{H}}{R}$ \\
\hline \multicolumn{3}{|c|}{ Độ cao chuẩn hạng I } \\
\hline 0.05 & 0.064 & 1.01 * $10^{-8}$ \\
\hline 0.04 & 0.057 & 8.90 * 10-9 \\
\hline 0.04 & Độ cao chuẩn hạng II \\
\hline \multicolumn{2}{|c|}{0.064} & 1.01 * $10^{-8}$ \\
\hline 0.03 & 0.057 & 9.17 * $10^{-9}$ \\
\hline
\end{tabular}


Từ bảng 1 chúng ta nhận thấy rằng để xây dựng hệ quy chiếu không gian quốc gia, chúng ta phải xác định được dị thường độ cao $\zeta$ của các điểm cơ sở của khung quy chiếu quốc gia với độ chính xác không thấp hơn $4 \mathrm{~cm}$ trong trường hợp đo nối thủy chuẩn hạng I từ các điểm độ cao hạng I quốc gia vào các điểm cơ sở và với độ chính xác không thấp hơn $3 \mathrm{~cm}$ trong trường hợp đo nối thủy chuẩn hạng II từ các điểm độ cao hạng II quốc gia vào các điểm cơ sở. Đây là các yêu cầu rất cao đối với việc xác định dị thường độ cao của các điểm cơ sở. Vấn đề khoa học được đặt ra trong bài báo này là nghiên cứu các biện pháp để xác định dị thường độ cao $\zeta$ của các điểm cơ sở của khung quy chiếu quốc gia với yêu cầu độ chính xác nêu trên.

\section{Giải quyết vấn đề}

Khi xây dựng hệ độ cao quốc gia dựa trên mặt Geoid cục bộ tại trạm nghiệm triều 0 (trạm nghiệm triều mà mặt biển trung bình tại đó được nhận làm mặt khởi tính cho hệ độ cao dựa trên mặt Geoid Gauss - Listing), dị thường độ cao độ chính xác cao của điểm cơ sở của hệ quy chiếu không gian quốc gia được xác định theo công thức (Hà Minh Hòa và nnk (2012a); Hà Minh Hòa (2012c)):

$$
\widetilde{\bar{\zeta}}=\frac{\hat{\bar{\zeta}}+\overline{\bar{\zeta}}}{2}
$$

ở đây dị thường độ cao GNSS/thủy chuẩn $\overline{\bar{\zeta}}=\bar{H}-H^{\gamma} ; \bar{H}$ - độ cao trắc địa của điểm được xác định từ kết quả xử lý dữ liệu đo GNSS trên điểm trong ITRF tương ứng với ellipsoid WGS84 quốc tế; $H^{\gamma}$ - độ cao chuẩn của điểm; dị thường độ cao trọng lực $\hat{\bar{\zeta}}=\bar{\zeta}+\frac{W_{0}-W_{0}}{10^{-5} \cdot \bar{\gamma}}$, thêm vào đó dị thường độ cao $\bar{\zeta}$ được xác định từ mô hình trọng trường Quả đất $\mathrm{EGM}, \overline{W_{0}}=62636856.0 \mathrm{~m}^{2} \cdot \mathrm{s}^{-2}$ - thế năng trọng trường thực của Geoid toàn cầu, $\mathrm{W}_{0}$ - thế năng trọng trường thực của Geoid cục bộ, $\bar{\gamma}$ - giá trị trung bình của gia tốc lực trọng trường chuẩn (đơn vị mGal) tại điểm.

Các dị thường độ cao $\overline{\bar{\zeta}}$ và $\hat{\bar{\zeta}}$ đều đã được chuyển về hệ triều 0 . Đối với lãnh thổ Việt Nam, thế năng trọng trường thực của Geoid cục bộ Hòn Dấu $W_{0}=62636847.2911 \mathrm{~m}^{2} \cdot \mathrm{s}^{-2}$ và trên toàn lãnh thổ Việt Nam biểu thức $\frac{\bar{W}_{0}-W_{0}}{10^{-5} \cdot \bar{\gamma}}=0.890 \mathrm{~m}$ (Hà Minh Hòa và nnk (2012a); Hà Minh Hòa (2012b); Hà Minh Hòa (2013b)). Khi đó,

$$
\hat{\bar{\zeta}}=\bar{\zeta}+0.890<m>\text {. }
$$

Bây giờ chúng ta khảo sát độ chính xác của dị thường độ cao GNSS/thủy chuẩn. Nếu chúng ta chỉ sử dụng công nghệ GPS, từ (2) suy ra đánh giá sai số trung phương độ caotrắc địa của điểm được xác định từ kết quả xử lý dữ liệu đo GNSS trên điểm trong ITRF tương ứng với ellipsoid WGS84 quốc tế $m_{\bar{H}}=0.0146-0.0158 \mathrm{~m}$. Nếu chúng ta xử lý 
đồng thời các dữ liệu đo GPS/GLONASS trên điểm trong ITRF, thì độ chính xác của độ cao trắc địa được nâng lên $\sqrt{2}$ lần, tức $m_{\bar{H}}=0.0103-0.0112 \mathrm{~cm}$ (Hà Minh Hòa, Nguyễn Ngọc Lâu (2011)). Như vậy, việc thu đồng thời tín hiệu từ các vệ tính GPS, GLONASS trên các điểm cơ sở của hệ quy chiếu không gian quốc gia và xử lý đồng thời các dữ liệu đo GPS/GLONASS trong ITRF là biện pháp đầu tiên để nâng cao độ chính xác của các dị thường độ cao của các điểm này. Giả thiết độ cao chuẩn của các điểm cơ sở là các độ cao chuẩn hạng $\mathrm{I}$, tức $m_{H^{\gamma}}=0.04 \mathrm{~m}$. Khi đó, sai số trung phương của dị thường độ cao GNSS thủy chuẩn hạng I được đánh giá bằng $m_{\overline{\bar{\zeta}}}=0.041 \mathrm{~m}$.

Vấn đề tiếp theo là đánh giá độ chính xác của dị thường độ cao trọng lực $\bar{\zeta}$ được xác định từ mô hình trọng trường Quả đất EGM2008. Theo các kết quả nghiên cứu trên 145 điểm độ cao hạng I trong tài liệu (Hà Minh Hòa và nnk (2012a)), khi coi các dị thường độ cao GNSS/thủy chuẩn hạng I không có sai số và đánh giá độ chính xác của các dị thường độ cao trọng lực $\bar{\zeta}$ được xác định từ mô hình trọng trường Quả đất EGM2008. chúng ta xác định được rằng sai số trung phương của các hiệu dị thường độ cao trọng lực và dị thường độ cao GNSS/thủy chuẩn hạng I được đánh giá bằng $m_{\overline{\bar{\zeta}}}=\sqrt{\frac{3.075082}{145}}= \pm 0.145 \mathrm{~m}$. Như vậy, trên lãnh thổ Việt Nam, dị thường độ cao trọng lực từ mô hình EGM2008 có sai số trung phương khá lớn. Các kết quả nghiên cứu này cũng trùng hợp với các kết quả nghiên cứu ở các nước mà ở đó các dữ liệu đo trọng lực chi tiết chưa được sử dụng để xác định các hệ số khai triển điều hòa của mô hình EGM2008.

Theo các kết quả nghiên cứu trong (Gomaa M. Dawod, Hoda F. Mohamed, Sherine S. Ismail. (2010)), việc so sánh các kết quả xác định dị thường độ cao theo phương pháp GPS/thủy chuẩn trên 207 điểm và dị thường độ cao từ mô hình EGM2008 tại thung lũng Bắc sông Nil và đồng bằng ở $\mathrm{Ai}$ Cập cho thấy sai số trung phương các độ chênh của các dị thường độ cao bằng $\pm 0.187 \mathrm{~m}$.

Theo các kết quả nghiên cứu trong (Ibrahim Yilmaz, Mustafa Yilmaz, Mevlut Gullu, Bayram Turgut. (2010)), dựa trên 313 điểm GPS/thủy chuẩn thuộc mạng lưới GPS cơ sở quốc gia Thổ Nhĩ Kỳ (khoảng 600 điểm) thực hiện so sánh dị thường độ cao GPS/thủy chuẩn với dị thường độ cao trọng lực được xác định từ mô hình EGM2008 cho thấy hiệu trung bình của dị thường độ cao GPS/thủy chuẩn và dị thường độ cao trọng lực được xác định từ mô hình EGM2008 ở mức $\pm 0.294 \mathrm{~m}$.

Như vậy, biện pháp thứ hai cần được triển khai để nâng cao độ chính xác của mô hình dị thường độ cao quốc gia là sử dụng các dữ liệu đo trọng lực chi tiết trên lãnh thổ quốc gia để hiệu chỉnh các hệ số khai triển điều hòa của mô hình EGM2008 theo công thức 
(Simberov B.P. (1975)):

$$
g=a_{00}+\sum_{n=2 m=0}^{\infty} \sum_{n m}^{n}\left(\bar{A}_{n} \cdot \cos m \lambda+\bar{B}_{n m} \cdot \sin m \lambda\right) \bar{P}_{n m}(\theta),
$$

Ở đây $\bar{P}_{m n}(\cos \theta)$ - hàm Legendre được chuẩn hóa; $\theta=90^{\circ}-\varphi ; \varphi, \lambda, H^{\gamma}, \gamma \quad$ - vĩ độ, kinh độ, độ cao chuẩn và gia tốc lực trọng trường chuẩn của điểm; $\bar{P}_{n m}(\theta)=q_{n m} \cdot P_{n m}(\theta)$ hàm dẫn xuất Legendre được chuẩn hóa, thêm vào đó

$$
P_{n m}(\theta)=\sin ^{m} \theta \cdot \frac{d^{m} P_{n}(\cos \theta)}{(d \cos \theta)^{m}}, q_{n m}=\sqrt{2 \cdot \frac{(n-m) !}{(n+m) !}} ;
$$

các hệ số khai triển hàm cầu được

chuẩn hóa

$$
\overline{\mathrm{A}}_{\mathrm{nm}}=\gamma \cdot(n-1) \cdot \Delta \bar{C}_{n m} ; \overline{\mathrm{B}}_{\mathrm{nm}}=\gamma \cdot(n-1) \cdot \bar{S}_{n m},
$$

$\bar{C}_{n m} ; \bar{S}_{n m}$ - các hệ số khai triển hàm cầu được chuẩn hóa của thế năng lực trọng trường của mô hình trọng trường Quả đất; $a_{00}=\gamma_{0}-\frac{\delta \gamma}{\delta H}$. $H^{\gamma}$, thêm vào đó

$$
\gamma_{0}=\bar{A}_{00} \cdot \bar{P}_{0}(\cos \theta)+\bar{A}_{20} \cdot \bar{P}_{2}(\cos \theta)+\bar{A}_{40} \cdot \bar{P}_{4}(\cos \theta) \quad \text { - gia tốc lực trọng trường chuẩn trên }
$$
mặt ellipsoid; $\frac{\delta \gamma}{\delta H}$ - gradient gia tốc lực trọng trường chuẩn.

Ngoài ra, biện pháp thứ ba là bình sai mạng lưới độ cao hạng I, II quốc gia dựa trên mặt Geoid cục bộ Hòn Dấu với các trị đo là các hiệu các đại lượng địa thế năng giữa các mốc độ cao. Khi đó, chúng ta sẽ nhận được các giá trị thế trọng trường thực $\mathrm{W}$ của các mốc độ cao hạng I, II quốc gia. Đây là nguồn dữ liệu bổ sung rất quý giá, độc lập với các dữ liệu đo trọng lực chi tiết, để hiệu chỉnh các hệ số khai triển điều hòa của mô hình EGM2008 theo công thức:

$$
\begin{aligned}
& \mathrm{W}(\rho, \theta, L)=\frac{f M}{\rho} \cdot\left\{1+\sum_{n=2}^{\infty}\left(\frac{a_{e}}{\rho}\right)^{n}\left[\sum_{k=0}^{n}\left(\bar{C}_{n k} \cdot \cos (k L)+\bar{S}_{n k} \cdot \sin (k \lambda)\right) \cdot P_{n k}(\theta)\right\}+\right. \\
& +\frac{\omega^{2}}{2} \rho^{2} \cdot \operatorname{Sin}^{2} \theta
\end{aligned}
$$

ở đây $\theta=90^{\circ}-B$.

Nếu dị thường độ cao trọng lực $\hat{\overline{\bar{\zeta}}}=\bar{\zeta}+\frac{\overline{W_{0}}-W_{0}}{10^{-5} \cdot \bar{\gamma}}$ có độ chính xác cao hơn $0.075 \mathrm{~m}$, thì với $m_{\overline{\bar{\zeta}}}=0.075 \mathrm{~m}$, chúng ta có đánh giá độ chính xác của dị thường độ cao $\widetilde{\overline{\bar{\zeta}}}(4.21)$ như sau:

- Đối với trường hợp độ cao chuẩn của các điểm cơ sở là các độ cao chuẩn hạng I: 


$$
m_{\overline{\bar{\zeta}}}=\frac{1}{2} \cdot \sqrt{m_{\overline{\bar{\zeta}}}^{2}+m_{\overline{\bar{\zeta}}}^{2}}=\frac{1}{2} \cdot \sqrt{(0.041)^{2}+(0.075)^{2}}=0.043 \mathrm{~m} ;
$$

- Đối với trường hợp độ cao chuấn của các điểm cơ sở là các độ cao chuấn hạng II:

$$
m_{\overline{\bar{\zeta}}}=\frac{1}{2} \cdot \sqrt{m_{\overline{\bar{\zeta}}}^{2}+m_{\overline{\bar{\zeta}}}^{2}}=\frac{1}{2} \cdot \sqrt{(0.051)^{2}+(0.075)^{2}}=0.045 \mathrm{~m} ;
$$

Như vậy, việc làm chính xác hóa các hệ số khai triển điều hòa của mô hình EGM2008 để nhận được dị thường độ cao trọng lực với độ chính xác cao hơn $0.075 \mathrm{~m}$ là công việc rất quan trọng trong quá trình xây dựng hệ quy chiếu không gian quốc gia.

Giả thiết rằng di thường độ cao $\widetilde{\bar{\zeta}}(3)$ được chuyển từ ITRF tương ứng với ellipsoid WGS84 về giá trị $\widetilde{\zeta}$ trong hệ quy chiëu không gian quốc gia tương ứng với ellipsoid quy chiếu quốc gia khổng có sai số. Khi đó, độ cao trắc địa $H$ của điểm cơ sở trong hệ quy chiếu không gian quốc gia có sai số trung phương

$$
m_{H}=\sqrt{m_{H^{\gamma}}^{2}+m_{\widetilde{\zeta}}^{2}}=\sqrt{(0.04)^{2}+(0.045)^{2}}=0.060 \mathrm{~m} .
$$

Khi chọn các độ cao trắc địa $H$ là các giá trị gần đúng của các độ cao trắc địa tương ứng với các điểm cơ sở của hệ quy chiếu không gian quốc gia, thực hiện bình sai ghép nối mạng lưới GNSS về hệ quy chiếu không gian quốc gia theo mô hình (Hà Minh Hòa (2013a)):

$$
\begin{array}{ll}
\bar{V}_{t}=d \tau_{1}^{k x 1} & , \bar{P}_{t}=\mu_{S}^{2} \cdot K_{t}^{-1}, \\
\bar{V}_{S}^{k x 1}=d \tau^{k x 1}-G_{S} . \Delta W+L_{S}, & \bar{P}_{S}=R_{S}^{e}
\end{array}
$$

ở đây $R_{S}^{e}$ - ma trận chuẩn nhận được khi bình sai mạng lưới GNSS trong ITRF, $\mathrm{K}_{\mathrm{t}}$ - ma trận tương quan của các tọa độ ellipsoid $\mathrm{B}, \mathrm{L}, \mathrm{H}$ của các điểm chung trong hệ quy chiếu không gian quốc gia, vectơ số hạng tự do

$$
L_{S}=\left(\begin{array}{c}
t-\tau_{1} \\
\cdots \ldots . . \\
0
\end{array}\right)
$$

$\mathrm{t}$ - vectơ các tọa độ ellipsoid của các điểm GNSS bậc $\mathrm{k} \times 1$, ở đây $\mathrm{k}=3 . \mathrm{N}$, còn $\mathrm{N}$ là tổng số điểm GNSS được bình sai trong ITRF, thêm vào đó các tọa độ ellipsoid địa $B, L, H$ của các điểm tương ứng với các điểm chung được sắp xếp ở các vị trí đầu tiên và các tọa độ này đã được chuyển về hệ quy chiếu không gian quốc gia theo các giá trị gần đúng của 7 tham số của mô hình Bursa-Wolf; $\tau_{1}$ - vectơ các tọa độ ellipsoid $B, L, H$ của các điểm chung bậc $\mathrm{k}_{1} \times 1$ trong VN2000 3D, ở đây $\mathrm{k}_{1}=3 . \mathrm{N}_{1}$, còn $\mathrm{N}_{1}$ là tổng số các điểm chung; ma trận $\mathrm{G}_{\mathrm{S}}$ có dạng

$$
G_{S^{\prime}}=\left[\begin{array}{llll}
G_{1} & G_{2}: & \ldots & \vdots \\
G_{N}
\end{array}\right]^{T}
$$


thêm vào đó khối $G_{i}(i=\overline{1, N})$ có dạng:

$$
G_{i}=\left[\begin{array}{ccc}
-\frac{\sin B_{i} \cdot \cos L_{i}}{M_{i}+H_{i}} & -\frac{\sin B_{i} \cdot \sin L_{i}}{M_{i}+H_{i}} & \frac{\cos B_{i}}{M_{i}+H_{i}} \\
\frac{-\sec B_{i} \cdot \sin L_{i}}{N_{i}+H_{i}} & \frac{\sec B_{i} \cdot \cos L_{i}}{N_{i}+H_{i}} & 0 \\
\cos B_{i} \cdot \cos L_{i} & \cos B_{i} \cdot \sin L_{i} & \sin B_{i}
\end{array}\right],
$$

còn vectơ $\Delta \mathrm{W}=\left[\begin{array}{lll}\delta_{X_{0}} & \delta_{Y_{0}} & \delta_{Z_{0}}\end{array}\right] \quad$ - vectơ các sai số hệ thống còn lại trong các tọa độ ellipsoid được gây ra do các sai số trong 7 tham số chuyển đổi tọa độ của mô hình Bursa - Wolf.

Sau khi bình sai ghép nối mạng lưới GNSS vào hệ quy chiếu không gian quốc gia, độ chính xác của độ cao trắc địa sẽ được nâng lên $\sqrt{2}$ lần, tức $m_{\overparen{H}}=\frac{0.060 \mathrm{~cm}}{\sqrt{2}}=0.042 \mathrm{~m}$.

Với $R=6356752.314 \mathrm{~m}$, sai số tương đối của độ cao trắc địa của điểm cơ sở thuộc hệ quy chiếu không gian quốc gia sẽ đạt mức $\frac{m_{\widetilde{H}}}{R}=\frac{0.042}{6356752.314}=6.61 * 10^{-9}$.

\section{Kết luận}

Việc xây dựng hệ quy chiếu không gian quốc gia là công việc rất phức tạp và gắn kết chặt chẽ với việc giải quyết bài toán xây dựng mô hình Quasigeoid quốc gia độ chính xác cao. Về phần mình, việc đạt được mô hình Quasigeoid quốc gia độ chính xác cao đòi hỏi phải giải quyết hàng loạt bài toán khoa học - kỹ thuật phức tạp:

- Lựa chọn các điểm thiên văn - trắc địa hạng I, II quốc gia làm các điểm cơ sở của hệ quy chiếu không gian quốc gia. Xây dựng mạng lưới GNSS dựa trên các điểm cơ sở nêu trên cùng với việc thu đồng thời các tín hiệu từ các vệ tinh GPS, GLONASS và xử lý đồng thời các dữ liệu đo GPS/GLONASS trong ITRF tương ứng với ellipsoid WGS84 quốc tế;

- Hiệu chỉnh các hệ số khai triển điều hòa của mô hình trọng trường Quả đất EGM dựa trên các dữ liệu đo trọng lực chi tiết trên lãnh thổ quốc gia và các nước lân cận (nếu có);

- Bình sai mạng lưới độ cao hạng I, II quốc gia dựa trên mặt Geoid cục bộ với các trị đo là các hiệu các đại lượng địa thế năng giữa các mốc độ cao. Hiệu chỉnh các hệ số khai triển điều hòa của mô hình trọng trường Quả đất $E G M$ dựa trên các giá trị thế trọng trường thực của các mốc độ cao hạng I, II quốc gia;

- Xác định dị thường độ cao của các điểm cơ sở của hệ quy chiếu không gian quốc gia theo công thức (3); 
- Bình sai ghép nối mạng lưới GNSS vào hệ quy chiếu không gian quốc gia.

Như vậy, để xây dựng hệ quy chiếu không gian quốc gia trong tương lai, ngay từ bây giờ chúng ta phải thực hiện nhiều biện pháp đồng thời, trong đo cấp bách nhất là xây dựng các phương pháp hiệu chỉnh các hệ số khai triển điều hòa của mô hình trọng trường Quả đất EGM dựa trên các dữ liệu đo trọng lực chi tiết và các giá trị thế trọng trường thực của các mốc độ cao hạng I, II quốc gia. Đồng thời tiến hành bình sai mạng lưới độ cao hạng I, II quốc gia dựa trên mặt Geoid cục bộ với các trị đo là các hiệu các đại lượng địa thế năng giữa các mốc độ cao. $O$

\section{Tài liệu tham khảo}

[1]. Augath W., Ihde J. (2002). Definition and realization of Vertical Reference System the European Solution EVRS/EVRF2000. FIG XXII International Congress, Wáhiton D.C., April 19-26 2002.

[2]. Gomaa M. Dawod, Hoda F. Mohamed, and Sherine S. Ismail. (2010). Evaluation and Adaptation of the EGM2008 Geopotential Model along the Northern Nile Valley, Egypt: Case Study. J. Surv. Engrg. Volume 136, Issue 1, pp. 36-40 (February 2010).

[3]. Hà Minh Hòa, Đặng Hùng Võ, Phạm Hoàng Lân, Nguyễn Ngọc Lâu (2005). Nghiên cứu cơ sở khoa học của việc xây dựng mạng lưới GPS các cấp hạng trong Hệ tọa độ động học. Báo cáo Tổng kết khoa học và kỹ thuật đề tài nghiên cứu khoa học công nghệ cấp Bộ Tài nguyên và Môi trường giai đoạn 2002 - 2004. Bộ Tài nguyên và Môi trường. Hà Nội 2005.

[4]. Hà Minh Hòa, Nguyễn Ngọc Lâu (2011). Vai trò của việc xử lý đồng thời các dữ liệu đo GPS/GLONASS trong ITRF để xác định dị thường độ cao độ chính xác cao. Tạp chí Khoa học Đo đạc và Bản đồ, No8, thánh 6 - 2011, trg. 1-6.

[5]. Hà Minh Hòa và nnk (2012a). Nghiên cứu cơ sở khoa học của việc hoàn thiện hệ độ cao gắn liền với việc xây dựng hệ tọa độ động lực quốc gia. Đề tài khoa học và công nghệ cấp Bộ Tài nguyên và Môi trường. Hà Nội - 2012.

[6]. Hà Minh Hòa (2012b). Nghiên cứu xác định thế năng trọng trường thực $W_{0}$ của mặt Geoid cục bộ trùng với mặt biển trung bình tại trạm nghiệm triều Hòn Dấu. Báo cáo khoa học. Tuyển tập báo cáo Hội nghị Khoa học và Công nghệ "Trắc địa và Bản đồ vì sự nghiệp Tài nguyên và Môi trường". Viện Khoa học Đo đạc và Bản đồ - Hội Trắc địa, Bản đồ và Viễn thám Việt Nam. Hà Nội - Tháng 10/2012. Trg. 6-19.

[7]. Hà Minh Hòa (2012c). Khả năng nâng cao độ chính xác xác định dị thường độ cao trên điểm GPS/thủy chuẩn nhờ Hệ độ cao dựa trên mặt Geoid cục bộ Hòn Dấu. Tạp chí Khoa học Đo đạc và Bản đồ, số 14, thàng 12-2012, trg.:1-7.

[8]. Hà Minh Hòa (2013a). Phương pháp bình sai truy hồi với phép biến đổi xoay. NXB 
Khoa học và Kỹ thuật, 287 trg. Hà Nội - 2013.

[9]. Hà Minh Hòa (2013b). Estimating the geopotential value $W_{0}$ of the local geoid based on data from local and global normal heights of GPS/Leveling points in Vietnam. Geodesy and Cartography, Taylor \& Francis, UDK 528.21, doi:10.3846/20296991.2013.823705, V.39: 99-105.

[10]. Ibrahim Yilmaz, Mustafa Yilmaz, Mevlut Gullu, Bayram Turgut. (2010). Evaluation of recent global geopotential models based on GPS/levelling data over Afyonkarahisar (Turkey). Scientific Research and Essays. Vol. 5(5), pp. 484-493, 4 March 2010.

[11]. Ihde J., Bruyninx C. (2008). Developments of the EUREF GNSS Services and Reference Networks. ICG-03 Meeting, 8-12 December 2008, Pasadena, USA.

[12]. Sánchez L., Martisnez W. (2001). Approach to the New Vertical Reference System for Colombia. In: Drewes H., Dodson A., Fortes L.P.S., Sánchez L., Sandoval P. Vertical Reference Systems. International Association of Geodesy Symposia, Volume 124, IAG Symposium Cartagena, Colombia, February 20-23, 2001. Springer.

[13]. Simberov B.P. (1975). Lý thuyết hình dạng Qủa đất. Matxcơva, Nedra, 432 trg. (Tiếng Nga). $\mathrm{O}$

\section{Summary}

Techno - scientific problems related to construction of state spatial reference system

Assoc. Prof. Dr. Sc. Ha Minh Hoa

Vietnam Institute of Geodesy and Cartography

This scientific article reserches a key problem of the construction of state spatial reference system - that's a construction of the high accurate state Quasigeoid model. Solving of the abovementioned problem is related to series of such complicated techno-scientific tasks as construction of state GNSS network based on both GPs and GLONASS technologies, adjustment of the first and second orders state levelling networks in local geoid based vertical system Hon Dau for determination of geopotentials of the first and second orders height points, correction of the harmonic expansion coefficients of the Earth Gravitational Mogel (EGM) based detailed gravimetric data on state territory and geopotentials of the first and second orders height points, adjustment and combining of GNSS network inti state spatial reference system. This articale also shows that the correction of the harmonic expansion coefficients of the Earth Gravitational Mogel (EGM) for getting of height anomaly with accuracy more than of $0.08 \mathrm{~m}$ is one of inportant scientific bases of the construction of the state Quasigeoid model with accuracy more than of $0.04 \mathrm{~m}$. O

Ngày nhận bài: 07/10/2013. 